\title{
IMPORTANCIA DEL ENFOQUE TRANSFORMADOR DE LA EVALUACIÓN COMO EJERCICIO PARA ORIENTAR LA CONSTRUCCIÓN DE POLÍTICAS PÚBLICAS
}

\section{IMPORTANCE OF THE TRANSFORMATIVE APPROACH OF EVALUATION, AS AN EXERCISE TO ORIENT THE CONSTRUCTION OF PUBLIC POLICIES}

David Quesada Garcíal

\author{
Fecha de recepción: 14 de mayo de 2017 - Fecha de aceptación: 4 de julio de 2017
}

\begin{abstract}
Resumen
El propósito de este artículo es reflexionar sobre el papel de la evaluación en los procesos de construcción de políticas públicas en la sociedad contemporánea, ya que elabora una discusión teórica sobre elementos que se deben considerar de forma integral e interdependiente. Denotando desde la revisión realizada la importancia del papel transformador y de aprendizaje continuo de la acción que plantea los ejercicios evaluativos, de esta forma, se expone una propuesta de principios para canalizar y orientar la evaluación. En sus conclusiones se destaca el rol de la ciudadanía, la inclusión de la perspectiva de género, la transparencia y la ética, para la promoción de dicho proceso de forma situada, permanente y transformadora.

Palabras claves: Gestión Pública, Sociología, Ciencias Políticas, Enfoque Emergente, Agenda pública.
\end{abstract}

\begin{abstract}
The purpose of this paper is to clarify the role of evaluation in the processes of construction of public policies in contemporary society, since it elaborates a theoretical reflection on elements that must be considered in an integral and interdependent way. Denoting from the revision made the importance of the transforming role and the continuous learning of the action that the evaluation exercises pose, this way, it exposes a proposal of principles to channel and orient the evaluation. In its conclusions, the role of citizenship, the inclusion of the gender perspective, transparency and ethics are highlighted, in order to promote this process in a permanent, transformative way.
\end{abstract}

Key Words: Public Management, Sociology, Political Science, Emerging Approach, Public Agenda.

\section{Introducción}

El presente artículo, incorpora una revisión sobre líneas de orientación para comprender la importancia del rol transformador en la evaluación de políticas públicas. Para ello en su primera parte se elabora sobre la importancia de la gestión pública y la institucionalidad con énfasis hacia las personas, es decir, un sistema orientado para fortalecer capacidades, en su segunda parte, el trabajo se centra en una aproximación a las políticas públicas, sobre las interrogantes de ¿qué es una política pública?, ¿quiénes son sus jugadores y jugadoras?, ¿qué factores intervienen en la construcción de la agenda pública?

1 Universidad Técnica Nacional,dquesadag@utn.ac.cr 
y sus dificultades. En la tercera parte, la discusión se encamina en situar la evaluación como un ejercicio fundamental para el desarrollo del proceso de las políticas públicas, el eje que articula dicho apartado versa sobre ¿qué es la evaluación?, ¿cuál es su utilidad?, centrándose principalmente en la perspectiva transformadora de la evaluación, presentando interrogantes y criterios evaluativos, para finalizar este apartado con una propuesta de principios para orientar la construcción de políticas públicas, entre ellos: la participación real de las personas en procesos horizontales, la ética-rendición de cuentas, la inclusión de la perspectiva de género, el manejo transparente de los recursos, la implementación de enfoques integrales y la evaluación como práctica situada y constante para mejorar el accionar y los resultados.

\section{Gestión pública e institucionalidad}

Uno de los grandes desafíos y finalidades del Estado contemporáneo es generar inclusión social, mediante el desarrollo cultural, económico, político, de las personas habitantes, promoviendo el respeto por derechos humanos y su dignidad humana, con base en sus matrices socio-culturales, sus necesidades y problemas situados, para ello es necesario lograr una renovación institucional desde una gestión pública enfocada en la consecución de resultados eficientes, eficaces y transparentes, desde una clara orientación de políticas públicas con vocación hacia el bienestar y transformación:

[...] el bienestar de los ciudadanos depende de la capacidad productiva, institucional, organizativa y política que se construya para la satisfacción de las necesidades y para la potenciación de oportunidades, capacidades y derechos de los costarricenses. Para eso, se requiere de un crecimiento económico ambientalmente sostenible, inclusivo, capaz de vencer las asimetrías estructurales, como condiciones para garantizar la reproducción de la vida acorde a la identidad colectivamente gestada y las aspiraciones para la convivencia social. También, se demanda un sector público eficiente y eficaz, probo, transparente, responsable, capaz de irradiar una acción articulada y oportuna a través de bienes y servicios públicos de calidad. Este propósito nacional es el sentido central de la política pública (MIDEPLAN, 2014, p. 30).

Para reflexionar cómo se pueden lograr estas metas y objetivos, es oportuno señalar siguiendo a Gómez y Arango (2012) que es por medio de las instituciones, las cuales logran realizar un maridaje con las normas, reglas, percepciones de la ciudadanía en la sociedad, de esta forma, se da a lugar a un concepto mucho más amplio denominado como la institucionalidad, que no solo abarca los organismos operativos del Estado sino "todo el conjunto de ideas, creencias, valores, principios, representaciones colectivas, formas y estructuras, relaciones interpersonales inter grupales que condicionan el comportamiento de los miembros de una sociedad estructurándola y caracterizándola en su dinamismo histórico” Lozano 1996 (citado en Correa, 1998, p. 87). Es decir, es un concepto que engloba y va más allá de la consideración e idea restringida de instituciones igual a Estado. 
Figura 1

Institucionalidad

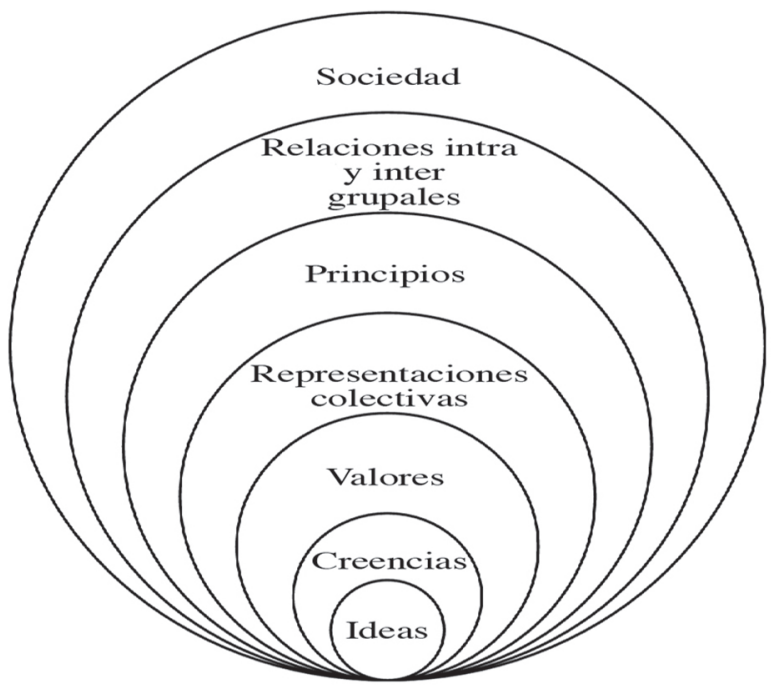

Elaboración propia 2017 a partir de Correa (1987).

Los componentes representados en la figura $n^{\circ} 1$, están en una estrecha relación de interdependencia con el contexto y dinamismo histórico de la sociedad, que caracteriza y estructura la forma en que los elementos societales se comportan, se articulan de acuerdo a necesidades; y a la forma en cómo estas se solucionan.

Uno de los procesos más importantes, sólidos y relevantes para lograr vincular las diversas necesidades e intereses de la población, a través de las relaciones inter e intra grupales, los principios, las representaciones colectivas, los valores, las creencias de la sociedad, en la finalidad de buscar la equidad social, el bienestar, estabilidad y la armonía entre sectores es "la administración pública" siguiendo lo planteado por (Gómez y Arango, 2012, p. 109), por tanto, es fundamental una alta calidad de gestión pública, que pueda incidir positivamente en la realización de las metas del Estado.

\footnotetext{
La gestión pública no es el confinamiento operativo de los Estados, sino el conjunto de capacidades que permiten traducir en situaciones factuales los propósitos que dan vida a las políticas y los programas de naturaleza gubernamental pero que tienen incidencia directa en la esfera de lo público [...] El alcance de la gestión pública está dado por la relación directa entre la sociedad y el Estado, los ciudadanos y el gobierno y las demandas ciudadanas y las políticas públicas (Uvalle, 2002, p. 4).
}

Lo anterior, puede fomentar procesos de mejora de gobernabilidad en el sentido de lo esbozado por Mayorga (2007) que explicita que este concepto se basa en un el equilibrio entre el nivel de las demandas societales y la capacidad del sistema político y sus instituciones, para poder responder ante estas demandas, es decir, cuando el Estado demuestra capacidad de acción para poder responder a dichas situaciones de forma eficaz y transparente, a través de la gestión pública.

La administración pública, funge según Uvalle (2002) como un sistema que produce y reproduce capacidades orientadas hacia las políticas públicas, de esta forma se pueden considerar a la gestión pública, como la gestión del gobierno, por consiguiente, "el modo de obrar que permite articular decisiones, capacidades, recursos, estrategias, programas, operadores y acciones que se encaminan 
a la exitosa gestión de las políticas públicas" (Uvalle, 2002, p. 2), por tanto, es fundamental que una de las funciones de la gestión pública sea fortalecer capacidades, para la consecución de políticas públicas responsables, exitosas ${ }^{2}$.

\section{Acercamiento a las políticas públicas: jugadores y jugadoras}

Las políticas públicas según Smith (2005) existen para solucionar los problemas que están presentes en la sociedad, los cuales inciden negativamente en las personas, grupos y comunidades, además, son definidas como una fuente instrumental, según Abarca, (2002) sobre el cómo estructurar acciones que conduzcan a la solución de los problemas públicos; desde un enfoque trans- disciplinar, que abra posibilidades de abordar y enriquecer la solución de los problemas públicos, articulados con la política para lograr así soluciones de problemas desde diversas acciones gubernamentales.

La formulación de las políticas públicas, está sujeta a la consideración e interrogante de ¿qué es un problema? y al mismo tiempo de ¿qué no es un problema?, "como una actividad de resolución de problemas que tiene tres básicos componentes, el problema, la política y los jugadores” (Smith, 2005, p. 1) [Traducción propia].

Figura 2

Componentes de la formulación de Políticas Públicas

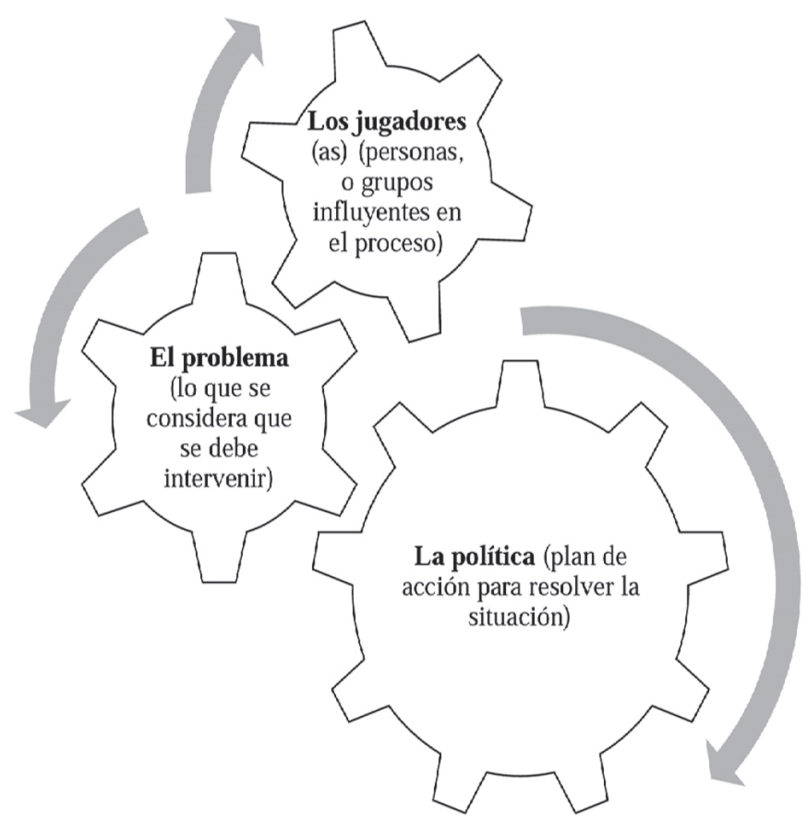

Elaboración propia a partir de Smith (2005).

2 "La aportación de la gestión pública a las instituciones consiste en que suministra los medios, los recursos y la capacidad instalada de los gobiernos para dar cumplimiento a las tareas de interés común. [...] La gestión pública, [...] es la encargada de ordenar, coordinar y producir los resultados que se encuentran en la agenda de las instituciones" (Uvalle, 2002, p. 5). 
Dichos componentes se sitúan en una relación de interdependencia y en un contexto determinado "la característica más importante de la actividad de formulación de políticas es que se trata de un proceso que ocurre en un contexto" (Smith, 2005, p. 1) [Traducción propia.], sumado a como lo presenta la figura $n^{\circ} 2$, cada engranaje puede tener un relativo peso mayor o menor, que los demás, aún así, dependen entre sí.

El proceso de considerar cuáles situaciones requiere intervención y su solución, es escogido por las y los elaboradores de política pública, en su formulación y construcción, para ser siguiendo a Nirenberg (2003) verdaderamente politizados o socialmente problematizados ${ }^{3}$ en las agendas públicas, ya que, esto se traduce en un reflejo de los valores que se establecen como prioritarios en la sociedad.

Figura 3

Campo de fuerzas en disputa en la formulación de políticas públicas

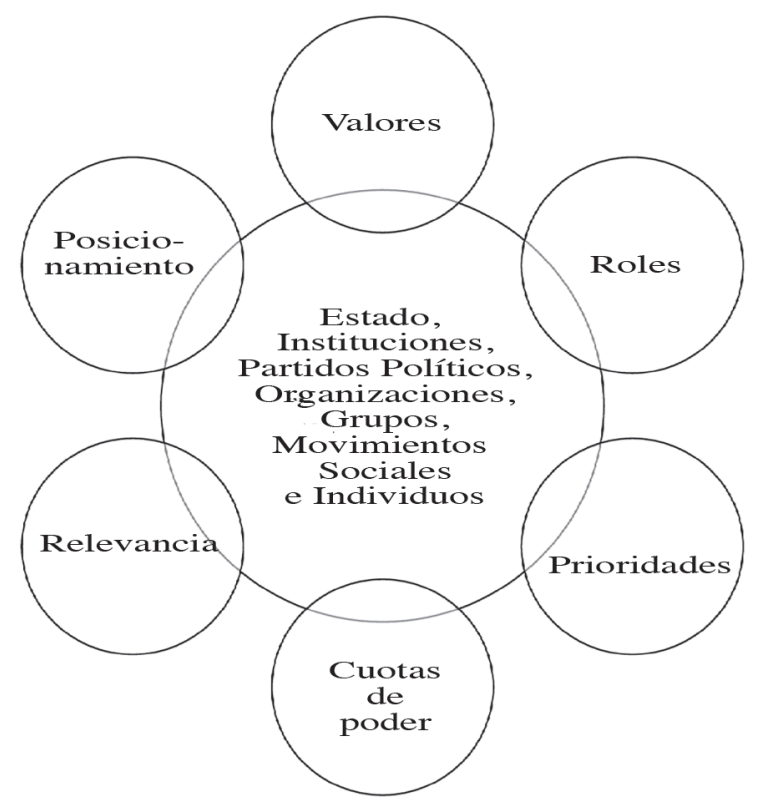

Elaboración propia a partir de Nirenberg (2003).

Como lo denota la figura $\mathrm{n}^{\circ} 3$, ¿de qué o quiénes dependen esa selección de cuestiones o a que corresponden a esos intereses?, la autora, refiere que es un complejo campo de fuerzas, que se encuentra en disputa a través de grupos, movimientos sociales, instituciones, partidos políticos, organizaciones e individuos, de acuerdo a su rol, relevancia, cuotas de poder y posicionamientos, puesto que, escogen, seleccionan temas (de acuerdo a sus prioridades), y de la misma forma, eligen su posible solución.

Es importante acotar, que las políticas públicas dependen del contexto en el que se desenvuelven, dado que, son construidas de acuerdo al dinamismo histórico en el que se encuentren la sociedad, entrando en juego en este proceso, la gobernanza, ya que es:

3 Se destaca que esta noción de las situaciones-problemas, y los jugadores "personas influyentes", se vincula con lo esbozado por Nirenberg (2003) sobre los intereses en disputa de grupos e individuos por establecer de acuerdo a sus cuotas de poder y posición, sus prioridades. 
Es un complejo de mecanismos, procesos, relaciones e instituciones por medio de los cuales los ciudadanos y los grupos articulan sus intereses, ejercen sus derechos y obligaciones y median sus diferencias [...] la idea central es que la gobernanza trasciende al Estado e incluye a las organizaciones de la sociedad civil y al sector privado (Serna, 2010, p. 36).

Ahora bien, ¿qué dificultades presenta para la gobernanza, la elaboración y toma de decisión en las políticas públicas? González (2002) reflexiona que la decisión sobre qué situaciones se colectivizarán, en torno a la selección o construcción de políticas públicas que harán frente a las problemáticas que la sociedad enfrenta es bastante complicado, debido a la existencia de una multiplicidad de intereses de cada sector y al carácter complejo de los problemas públicos y las limitantes de recursos, pero agrega que "la imposibilidad de la unanimidad y de la escasez de recursos públicos, no debe ser pretexto para prolongar problemas o evadir responsabilidades públicas” (González, 2002, p. 53), en síntesis, los retos en la elaboración de políticas públicas no deben ser una excusa, o un pretexto para rehuir y eludir su realización.

Aunado a que, las mismas están orientadas a generar y lograr cambios sobre las situaciones o necesidades politizadas en la sociedad, por tanto, la relevancia de decisiones de alta calidad, aunque se ha considerando también que "suele decirse que no tener política, es en sí una política, al dejar que las cosas continúen como están" (Nirenberg, 2003, p. 27).

\section{Aproximación a las políticas públicas: agenda pública}

Gerston (2010), plantea una analogía entre el clima y la agenda pública, ya que, "al igual que un barómetro que reacciona a diferentes condiciones climatológicas, la agenda pública cambia con el cambio de prioridades y valores públicos. Como tal, la agenda pública asume una presencia dinámica" (Gerston, 2010, p. 48) [Traducción propia], al establecer que esta se modifica con base en el contexto de prioridades públicas y valores de una determinada sociedad, caracterizadas como cambiantes y dinámicas.

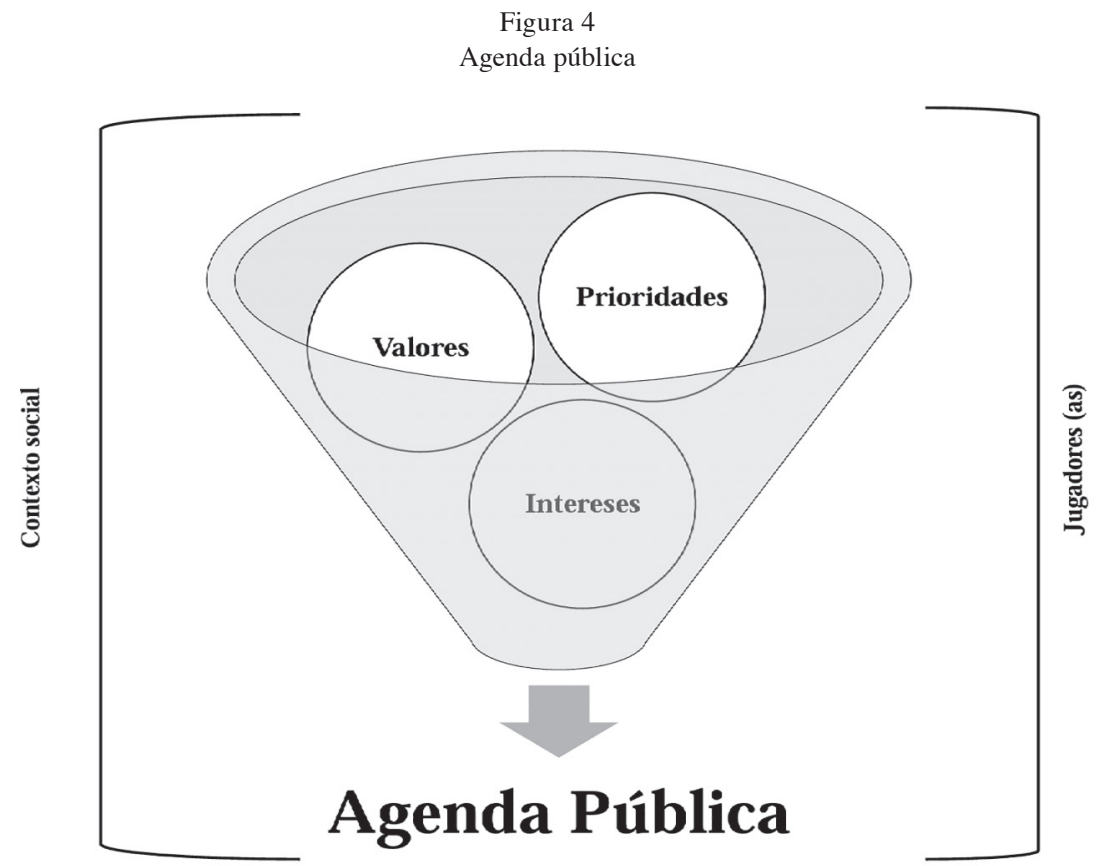

Elaboración propia a partir de Gerston (2010). 
La agenda pública depende de lo expresado en la figura $\mathrm{n}^{\circ} 3 \mathrm{y}$ de las cuotas de poder de las y los jugadores denotados en la figura $\mathrm{n}^{\circ} 2$. Por consiguiente, los problemas que son socialmente problematizados presentan características como las siguientes:

\footnotetext{
Algunos problemas son un fenómeno de “una vez": que llegan a la agenda pública, son resueltos por los que elaboran la política pública, y nunca vuelven a aparecer. Otros problemas son de carácter abierto: no están agravando suficiente para captar el apoyo, pero son lo suficientemente molestos para atraer la atención. A veces, un problema puede ser tan complicado o tan divisible que pequeños retazos de otras soluciones temporales pueden ser aplicados, dejando la impresión entre los participantes que el tema surgirá en otro momento (Gerston, 2010,p. 48). [Traducción propia]
}

De este modo, la agenda pública está compuesta por diferentes tipos de problemas, que se caracterizan por su dinamismo, volatilidad y complejidad, que requieren la acción y atención de las personas e instituciones que elaboran la política pública, consecuentemente, es fundamental en la construcción de cualquier política pública, su contextualización, de forma integral tanto en el tema de los problemas que se introducen en la agenda pública, así como los intereses, y perspectivas a considerar de las personas jugadoras, sobre las posibles soluciones a desarrollar.

La identificación de los problemas públicos requiere como se ha mencionado precisar el contexto de la situación y la visión de diversos actores, por lo tanto, es necesario los abordajes integrales y transdisciplinarios para conocer qué situaciones se priorizan, ¿por qué? y ¿qué actores influyen?, destacando que se debe articular la visión de gobernar de acuerdo a política pública, es decir, con sentido público, para según Aguilar (1992) lograr incorporar la opinión, la participación y la corresponsabilidad; para lograr en los grupos menos favorecidos:

Su poder de negociación, hasta un grado donde los (interventores) no puedan imponer unilateralmente sus condiciones y regulaciones sobre los pobres, como receptores pasivos, sino más bien que los términos y condiciones de colaboración sean el resultado de un proceso en el que ambas partes sean respetuosas de las prioridades y de los intereses específicos de cada quien. Verhagen (citado en Carmen, 2004, p. 72).

Consecuentemente, un problema público es un hecho socio-cultural sobre un problema inmerso dentro un sistema político específico, es decir, un asunto situado que responde a las necesidades e intereses que son priorizados y que exigen resolución o actuación, pero cada elemento debe ser respetuoso de las diferentes perspectivas, para la formulación de la agenda pública donde:

Conocimiento y juicio moral se combinan para que un fenómeno se transforme en problema y en desafío. Un problema público es socialmente asumido: se genera un proceso de responsabilización por parte de tal o cual segmento de la sociedad. Un grupo determinado decide que un problema es acreedor a una intervención pública porque dicho grupo tiene capacidad, autoridad para promoverlo (Ives y Thoening, 1994, p. 111).

Las políticas públicas también pueden definirse como "soluciones específicas de cómo manejar los asuntos públicos" (Lahera, 2004, p. 7), en un momento y espacio determinado, por consiguiente, se está en presencia de decisiones de las personas formuladoras de políticas públicas, en consonancia, González (2002) define a las políticas públicas bajo dos características fundamentales: 1- son una forma de expresión de las decisiones colectivas, y 2- surgen a través de una articulación de múltiples intereses y preferencias.

Es necesario presentar una definición más amplia de las políticas públicas, las cuales se configuran en "el conjunto de objetivos, decisiones y acciones que lleva a cabo un gobierno para solucionar los problemas que en un momento dado los ciudadanos y el propio gobierno consideran prioritarios" (Tamayo citado en Nirenberg, 2003, p. 23-24), ante esta definición es importante complementar, según 
Smith (2005) que no solo el gobierno ${ }^{4}$ es la única institución creadora de políticas públicas, sino que también surgen de "autoridades culturales" de diferentes ámbitos, como el religioso, educacionales y empresariales, entre otros.

\section{La evaluación como ejercicio fundamental para las políticas públicas}

La evaluación es una práctica fundamental para decidir y mejorar la acción por medio de la reflexión sobre lo realizado y/o lo que se pretende realizar, es decir, según Ulloa y Giomi (2002), distanciarse de lo que se hace o se hizo, para observar y analizar que obstáculos, facilidades y logros se han obtenido, para obtener una mejor direccionalidad, en este caso para las políticas públicas y sus consiguientes programas que derivan en diversos proyectos (sociales, culturales, económicos). La evaluación posee una gran variedad de definiciones, entre ella se tiene que es:

Una actividad programada de reflexión sobre la acción, basada en procedimientos sistemáticos de recolección, análisis e interpretación de información, con la finalidad de emitir juicios valorativos, fundamentados y comunicables sobre las actividades, resultados e impactos de proyectos o programas, y formular recomendaciones para tomar decisiones que permitan ajustar la decisión presente y mejorar la acción futura (Nirenberg et al, 2000, p. 32).

Las diversas nociones sobre la evaluación, según Vargas (2001) varían del contexto histórico y la posición en que se ubique la persona que investiga e incluso desde la ciencia desde la que se elabore, siendo principalmente tres los enfoques predominantes en materia de la evaluación, que dependen de la perspectiva sobre la naturaleza de la realidad, es decir, si se considera a la realidad como objetiva es neopositivista, en donde la evaluación es vista como descripción de la realidad, si se considera subjetiva es comprensivista, es decir, la evaluación como comprensión de la realidad y por último, si se considera a la realidad de acuerdo al enfoque transformador, la evaluación se ubica en el paradigma emergente de reflexión-praxis-acción, en donde la evaluación es posicionada como transformadora de la realidad.

Desde el enfoque de la evaluación como transformación, es oportuno describir las siguientes interrogantes, que se detallarán por medio de la figura $n^{\circ} 5$, para denotar ejemplos de interrogantes evaluativas, que deben ser considerados en los procesos de formulación, ejecución evaluación de las políticas públicas, programas, proyectos ${ }^{5}$ para lograr reflexividad.

$4 \quad$ Una definición complementaria de Políticas Públicas es la siguiente: “una de las actividades básicas y fundamentales del Estado se relaciona con la puesta en marcha de distintos tipos de programas, obras y actividades que un determinado momento pueden ser útiles a una comunidad concreta" (Kuschick, 2002, 105).

5 Un proyecto es entendido como "un conjunto de actividades que se proponen realizar de una manera articulada entre sí, con el fin de [...] resolver problemas, dentro de los límites de un presupuesto y de un periodo de tiempo escasos" (AnderEgg y Aguilar, 2004, p. 12). 
Figura 5

Interrogantes evaluativas

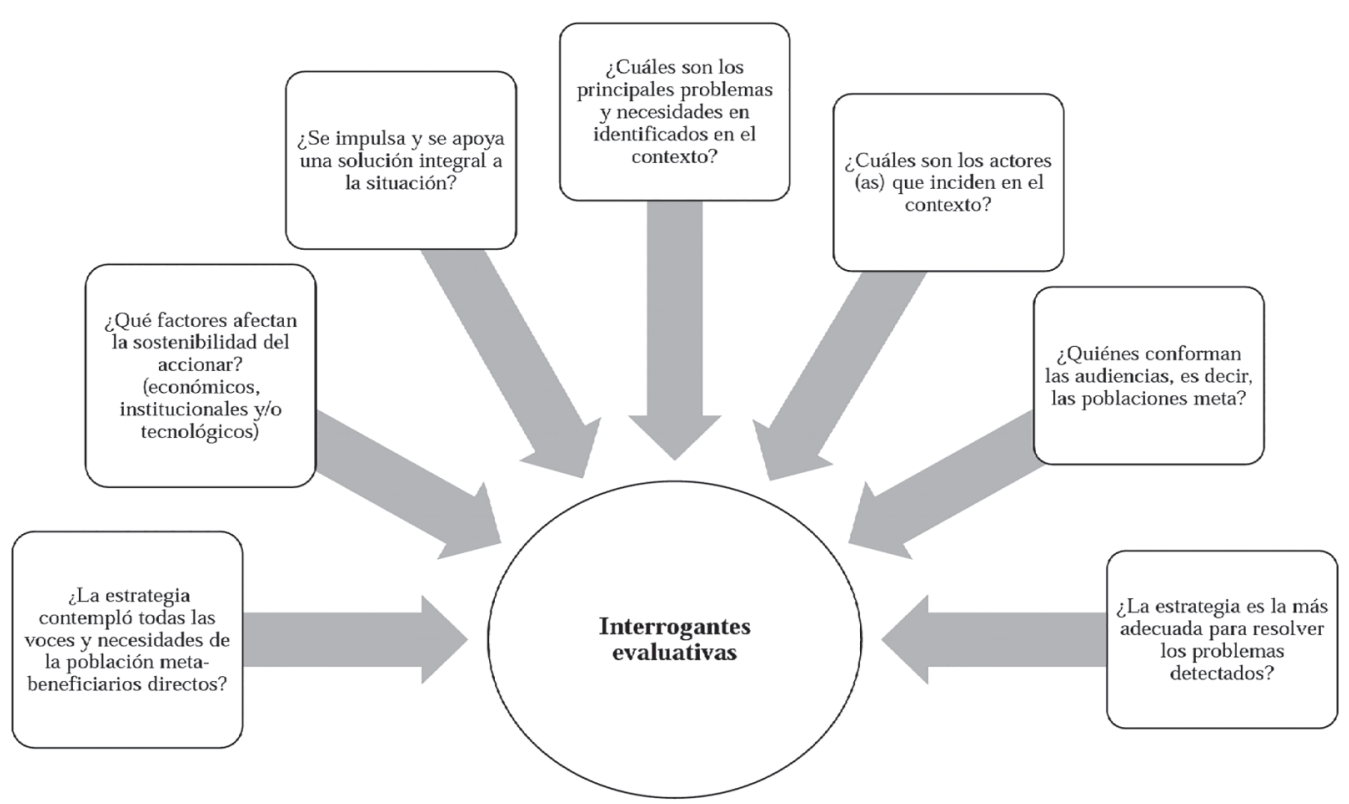

Elaboración propia (2017) a partir de Nirenberg (2013) y Picado (2002).

Por tanto, la figura $n^{\circ} 5$, ejemplifica a partir de las interrogantes que la evaluación como transformación consistirá en una reflexión constante, ya sea anterior a la acción, durante la acción y/o posterior a la acción, esto con la finalidad de poder tomar decisiones y realizar recomendaciones fundamentadas sobre las futuras acciones, obteniendo con esto una direccionalidad mejorada y centrada sobre las políticas públicas.

Dentro de la pedagogía crítica la evaluación se concibe como un proceso de autorreflexión y de producción de conocimiento que realizan las y los participantes sobre la teoría y la práctica de su propia acción. La evaluación se convierte así en praxis crítica a lo largo de todo el proceso, pero su interés no es únicamente reflexionar sino promover cambios, tanto en las personas como en lo evaluado. Surge así la reflexión-acción-reflexión en donde los conocimientos producidos mediante la evaluación se revierten a los propios actores del proceso, en la dinámica transformadora de su práctica, que provoca de nuevo la reflexión (Vargas, 2001, p. 41-42).

Lo anterior, parte del enfoque transformador, derivado del paradigma emergente, el cual no posiciona una separación entre la persona que evalúa y el mal llamado objeto de evaluación, ni en una mera descripción de la realidad, sino en un proceso de generación de conocimientos y reflexividad, entre las personas evaluadoras, el gobierno o institución ejecutora y las personas de los diferentes frentes de trabajo; es decir, los grupos meta a las que va dirigida la acción. Por tanto, desde este enfoque la evaluación se define como:

Un proceso de reflexión y análisis crítico mediante el cual se trabaja sobre una situación, problemática o proceso social, partiendo de valoraciones y concepciones iniciales que se van reformulando en el mismo proceso, [...] su interés no es únicamente reflexionar sino promover cambios, tanto en las personas como en lo evaluado (Vargas, 2001 p. 42). 
Se evidencia, la importancia que este enfoque le dota a la participación comprometida de todas las personas involucradas en el análisis de la acción diseñada, dado que, además "abandona la posición tecnocrática asumida en los dos enfoques anteriores (neo-positivista y comprensivista) [...] Los y las evaluadores se integran activamente con el resto de grupo, tratando de generar niveles superiores de toma de conciencia” (Vargas, 2001, p. 43) [paréntesis agregados], lo que elimina en cierta medida, la jerarquización de quienes tienen mayores cuotas de poder y de palabra para de tomar y decidir acciones, en el campo de disputa de las y los jugadores, fomentando una responsabilidad compartida, además, de un rol activo de la ciudadanía sobre la consulta, y solución de la forma en cómo resolver las situaciones.

\section{Criterios evaluativos rumbo al aprendizaje continuo}

Para todo ejercicio de evaluación se necesita elaborar y situar desde lo teórico y lo práctico, en tanto contexto de la situación, una serie de elementos o componentes básicos en forma de criterios de evaluación, para cimentar decisiones respaldadas, las cuales sirvan para tomar decisiones o emitir deliberaciones detalladas, analizadas y estudiadas a profundidad.

De esta forma, los criterios son los elementos con los que fundamente una decisión o se emite un juicio, para los ejercicios evaluativos, "el conjunto de elementos con que se juzga una situación y como el empleo de normas para conocer la verdad, también se define criterio como el elemento que se utiliza para fundamentar una decisión o emitir un juicio" (Picado, 2002, p. 9).

La siguiente figura denota ejemplos de criterios evaluativos para ser considerados en los procesos de formulación, ejecución y evaluación de las políticas públicas, programas ${ }^{6}$, proyectos.

Figura 6

Criterios evaluativos

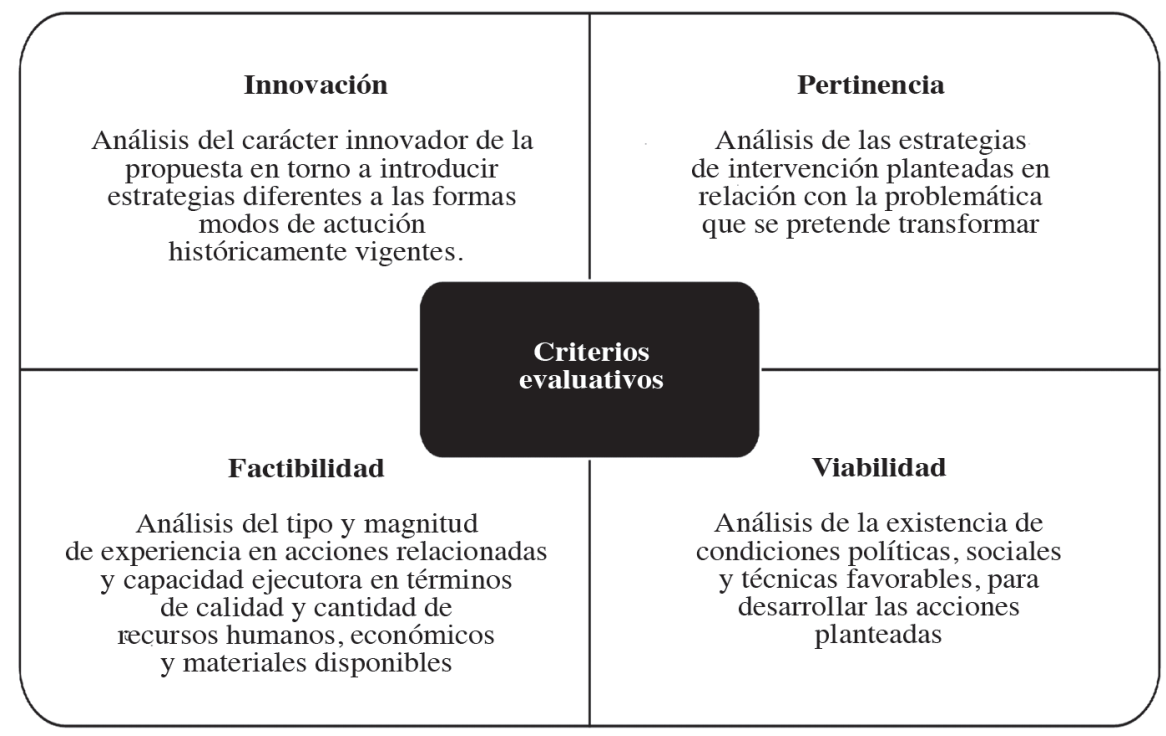

Elaboración propia a partir de Nirenberg (2003, p. 103-115) y Picado (2012).

6 Un programa es definido como "esfuerzos concretos, legales y burocráticos para convertir las intenciones de la política general en acciones gubernamentales específicas. [...] Un programa mezcla recursos gubernamentales, combinando autorización legal, los ingresos y el personal públicos en un paquete de actividades emprendidas por una organización pública particular" (Rose, 1999, p. 270). 
Los juicios anteriores, ejemplifican que los procesos evaluativos deben considerar criterios sólidos para la toma de decisión, por tanto, Gómez y Sainz (2001) caracterizan y definen a la evaluación como un proceso de evaluación y valoración continua, cuyo propósito es mejorar la gestión realizada de la intervención que se hace o se hizo, cuyos resultados, para ser útiles, deben ser comunicables a todas las personas, en plazos no prolongados que permitan ser aplicables a las recomendaciones; por tanto, prosiguen los autores, la evaluación sitúa un contenido de aprendizaje, es decir, aprender de lo que se realiza, al ser un proceso de retroalimentación, y finalmente, tiene una función de rendición de cuentas, para demostrar si lo ejecutado, obtuvo los resultados esperados.

Hablamos de evaluación cuando nuestra acción de valoración se centra en la comparación entre la situación que hemos alcanzado o estamos alcanzado y la situación de partida que dio origen a la acción. Se trata de establecer si esa situación de llegada guarda correspondencia con la estrategia de intervención que habíamos considerado apropiada y si los resultados son suficientes significativos para consolidarse y hacer desaparecer las condiciones identificadas inicialmente como negativas o como fuente de los problemas que tratamos resolver (Gómez y Sainz, 2001, p. 70-71).

Un componente central de esta definición es la comparación entre la situación que dio origen a los problemas y la acción que se planteó y ejecutó, para analizar si lo realizado mejoró o no esas condiciones iniciales o problemas presentes. Rivera (2002) por su parte, establece a la evaluación como un elemento indispensable en las sociedades, la caracteriza como pluralista, es decir, es portadora de visiones de mundo (neopositivismo, comprensivista y transformadora) y posiciones políticas particulares, pero aun así, no debe perder su carácter de bienestar social, debido a las exigencias de transparencia, rendición de cuentas de las gestiones públicas por parte de la ciudadanía para garantizar una correspondencia entre las acciones y los intereses sociales en forma de diálogo y crecimiento muto, además que conjuntamente:

La evaluación no debe ser confundida con el control de gestión y debe apoyarse sobre una ética cuidadosamente definida: su frecuencia y su continuidad no son las mismas, los responsables de la evaluación no deben ser perpetuados: el recurso a los profesionales exteriores debe ser promovido. Stoffaes (citado en Rivera, 2002, p. 19).

Agregando la importancia de considerar el contexto de la acción, ya que, según Rivera (2002) no es simplemente comparar objetivos sobre logros obtenidos, sino realizar una reconstrucción de la acción pública a partir de las y los actores que intervienen, en donde la ética es fundamental bajo la idea de la importancia de que quienes realicen la evaluación no sean los mismos que elaboran las políticas públicas.

Todo análisis y evaluación, que precede a la implementación de cualquier política pública, programa, proyecto es fundamental, para asegurar en cierta medida el cumplimiento de los resultados esperados y la transformación de la situación, dado que:

\footnotetext{
Se trata de establecer la pertinencia de la propuesta: la adecuación de las intervenciones que propone a la realidad que se pretende modificar; su coherencia interna en cuanto a la adecuación de recursos a actividades y de estas a metas y objetivos y su factibilidad, o sea, la capacidad de la institución ejecutora para llevarla a cabo en términos de la calidad y cantidad de recursos humanos, económicos y materiales disponibles, así como su viabilidad en función de la existencia de condiciones políticas, sociales y técnicas favorables, para desarrollar las acciones planteada (Nirenberg, 2003, p. 103-104).
}

Asimismo, este tipo de evaluación (ex ante) permite realizar un análisis de las posibilidades de formular y ejecutar las acciones futuras, donde su finalidad es siguiendo a Cohen (1988) proporcionar a las personas e instituciones de criterios para tomar la decisión si debe implementar la acción o no.

No obstante, los análisis en los procesos de evaluación deben contemplar interrogantes evaluativas, criterios de evaluación, no solo en el tipo de evaluación ex ante, sino en toda formulación, ejecución, monitoreo, de políticas públicas, dado que, estas orientarán y suministrarán una direccionalidad de 
mayor calidad para las y los tomadores de decisión y constructores de políticas públicas, del Estado en conjunto con la ciudadanía en el ejercicio, de acuerdo a la situación-problema y contexto de la sociedad. La evaluación es una práctica única debido a sus condicionantes, es decir el contexto, además, es un ejercicio fundamental para decidir y mejorar la acción por medio de un proceso de reflexividad sobre lo realizado y lo que se desea, para obtener resultados que transformen la situación:

La modalidad o modalidades de intervención propuesta se basan explícita o implícitamente en una concepción del cambio que supone que, si se llevan adelante ciertas acciones en determinadas circunstancias, se obtendrán resultados esperados, en términos de superar, contribuir a solucionar o evitar que empeoren determinados problemas sociales (Nirenberg, 2003, p. 114).

Por tanto, la evaluación busca obtener una direccionalidad deseada, por tanto, la evaluación consistirá en una actividad que precede ${ }^{7}$ (ex ante), se realiza durante y posterior a la acción (ex post), de acuerdo a los tipos que se seleccionen, para buscar poder tomar decisiones, realizar recomendaciones fundamentadas sobre futuras acciones.

\section{Propuesta de principios para orientar la evaluación de las políticas públicas desde el rol transformador}

A continuación, se presenta una serie de elementos a forma de principios para ser considerados en todo proceso de construcción de las políticas públicas, de forma integral, que denotan y promueven a la evaluación como un ejercicio orientado a la transformación.

1. La participación real de las personas, (rol activo de la ciudadanía) en los procesos de las políticas públicas

El rol activo de la ciudadanía es y debe ser posicionado como una forma de empoderamiento de las situaciones que se enfrentan, ya que, "sin participación, las personas no pueden apropiarse de las soluciones" (Costa, 2004, p. 4), lo anterior, procura una responsabilidad crítica y activa de diálogo con los diversos jugadores y jugadoras que intervienen en los procesos de construcción de políticas públicas, donde se debe considerar como fundamental la dimensión cultural de grupos específicos que han sido marginalizados, por ejemplo, población indígena, migrante, derechos de la comunidad LGTBQ, entre otros.

De lo contrario, sin un rol activo de las personas ${ }^{8}$, lo que se está fomentando en los procesos de políticas pública, es una falsa participación o lo que Raff Carmen (2004) definiría como "participulacion", donde la participación es usada como un concepto popular que le permitía a las y los planificadores lograr introducir cambios "sin hacer referencia seria a cambios estructurales" (Carmen, 2004, p. 59), por tanto, si las personas no son vistas como actores (as) fundamentales y activos desde posiciones horizontales, se seguiría reproduciendo una lógica que no alude a las situaciones estructurales que producen los problemas y necesidades, promoviendo soluciones temporales, enfrentando lo anterior al reto que se enmarca en que, es la ciudadanía la que debe apropiarse de su papel y re-conceptualizar su relación con el Estado, no sólo como garante de derechos, sino como depositario de deberes, para trabajar en conjunto, desde diversas posicionamientos y sectores en la formulación de soluciones.

7 Por ejemplo, la evaluación ex ante es retrospectiva a la acción y/o con foco en la formulación, debido a que se inician antes de tomar la decisión "el objetivo de verificar si cumple o no con las condiciones requeridas para su implementación" (Nirenberg et al, 2003, p. 103).

8 Se debe incluir de acuerdo a Nirenberg (2003) a los diversos actores, especialmente a los beneficiarios, en las diferentes etapas identificación de la problemática, decisión relativas al destino de los recursos. 
2. La inclusión de la perspectiva de género

En todo proceso de formulación, ejecución y evaluación de políticas públicas se debe considerar a la perspectiva de género, y facilitar "el conocimiento y ejercicio de los derechos de las mujeres, su acceso a recursos y procesos de toma de decisión" (Costa, 2004, p. 5) como elemento que transversalice y articule las iniciativas, entendiendo, que el enfoque de género no alude solamente a la idea simplista y reducida que la paridad de género es una igualdad de participación de $50 \%$ y $50 \%$ entre hombres y mujeres, sino que apunta a la consideración de que las mujeres enfrentan una serie de condiciones estructurales que limitan el desarrollo de sus potencialidades y capacidades, tales como la violencia de género, el lenguaje sexista, la misoginia, la imposición de los roles, y la definición de lo masculino en contraposición a lo femenino, donde se establecen relaciones de jerarquías de superioridad o inferioridad, todo lo anterior, desde el patriarcado como ente legitimador de la violencia. De esta forma, las políticas públicas deben considerar y trabajar articuladamente para generar soluciones integrales, ya que, las políticas públicas no pueden aplicarse o elaborarse sin considerarse estas condiciones estructurales.

3. La ética, la transparencia, rendición de cuentas y responsabilidad en el manejo transparente de los recursos

Dichas responsabilidades recaen en la importancia de explicar de forma clara y transparente el uso que se le dio a los recursos utilizados (económicos, estructurales, humanos), enmarcados como medio de control de las y los ciudadanos y rendición de cuentas [accountability], dado que, los recursos son limitados y escasos, y un manejo inadecuado de los recursos, conllevará a pobres resultados, eliminado así la mentalidad transformativa de las acciones.

Además, este principio busca mecanismos y acciones que "fortalecen la credibilidad, la confianza y finalmente la aceptación del gobierno y de la administración pública por parte de los ciudadanos" (Gómez y Arango, 2012, p. 112), siendo uno del aspecto más importante de las políticas públicas, el de atender problemas socialmente politizados, sobre necesidades de las personas, grupos, comunidades, por tanto, es un deber ético realizar este proceso de la mejor forma, con información simétrica en todas las partes que intervengan.

\section{La implementación de enfoques integrales}

La transversalización de las soluciones de las problemáticas o temas que son atendidos en las políticas públicas, deben partir de la "inclusión de enfoques amplios de problemática social, lejos de abordajes específicos que consideran los problemas de forma particular ${ }^{9}$ o fragmentada" (Nirenberg, 2003, p. 35). De esta forma, al realizar un análisis de los factores que intervienen en las problemáticas/ situaciones se debe enfocar en resolver y en darle una atención en todas sus dimensiones, y no solo en una pequeña parte de la situación. Además, se debe considerar que existen muchos factores contextuales que pueden influir sobre los resultados de las políticas públicas, coyunturas específicas e intereses de las y los jugadores.

\section{La evaluación constante, como práctica situada y fomentada}

Finalmente, se debe buscar generar políticas inteligentes que produzcan mejores resultados, es decir, que sean eficaces, eficientes, sostenibles en el tiempo, y coherentes, por medio, de "la evaluación

9 Ya que en contextos reciente se "comienza a prevalecer una lógica de implementación de políticas sociales acotadas y segmentadas que apuntan más a paliar algunas consecuencias de la privación material, cultural, y simbólica de los grupos vulnerables que a encarar los factores cáusales de la pobreza, particularmente los económicos” (Nirenberg, 2003, p. 27). 
como un elemento indispensable en las sociedades", (Rivera, 2002), debido a las exigencias de transparencia, y rendición de cuentas de las gestiones públicas por parte de la ciudadanía, que van encaminadas para garantizar una correspondencia entre las acciones y los intereses sociales en forma de diálogo y retroalimentación.

Figura 7

Principios orientadores en Políticas Públicas

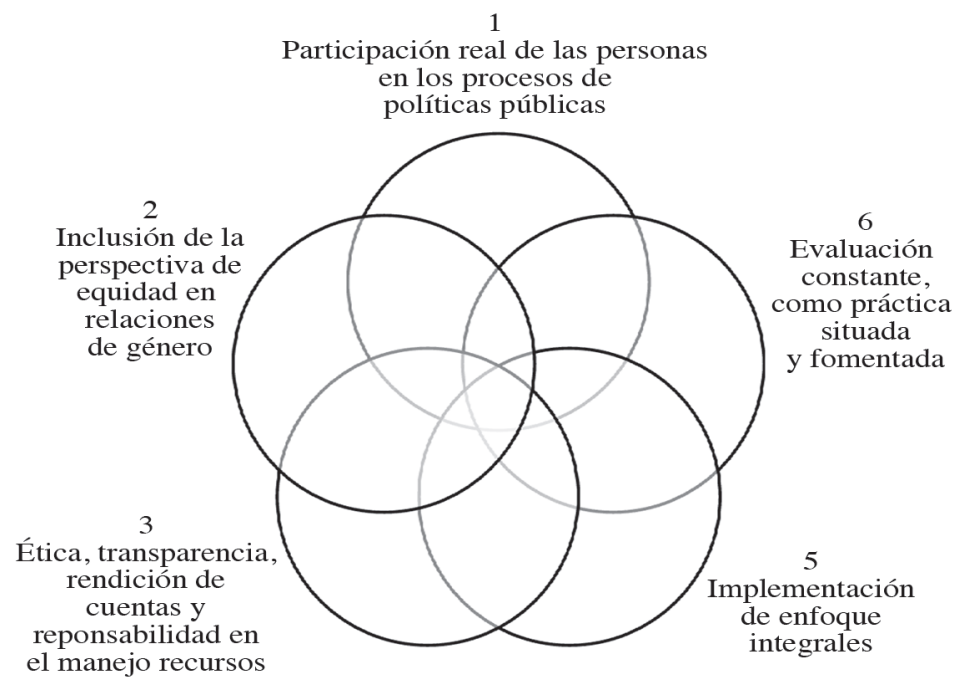

Elaboración propia, se retoman los aportes de Costa (2004), Carmen (2004), Gómez y Aranjo (2012), Nirenberg (2003) y Rivera (2002).

\section{Conclusiones}

La importancia de la evaluación desde el enfoque transformador se denota al ser un mecanismo que favorece procesos de orientación horizontales para mejorar las acciones realizadas y las soluciones de los problemas, al generar reflexividad en todos sus actores, a través de una praxis crítica de acciónreflexión-acción, como menciona (Vargas, 2001), para ello es fundamental la gestión pública, dado que, es la encargada de diseñar y ejecutar las estrategias del Estado, por tanto, es necesario el apoyo, el desarrollo, la renovación y el fortalecimiento humano, en el ámbito personal por medio de capacitaciones, estrategias, e investigaciones, desde los principios de eficiencia, eficacia y calidad que puedan mejorar las decisiones y acciones que son llevadas a cabo, al estar vinculadas con las necesidades y problemas de la población desde los principios éticos de transparencia y optimización del uso de los recursos, y el control de la ciudadanía.

Las políticas públicas son entendidas como las decisiones y acciones que realiza en este caso el Estado desde la gestión pública ${ }^{10}$ para resolver los problemas de la agenda pública. Por tanto, se percata la importancia que poseen estas orientaciones que son tomadas para responder a las situaciones

10 No obstante, es menester destacar que "todas las organizaciones, incluidas las públicas son inevitablemente imperfectas [...] Ninguna organización gubernamental puede satisfacer todos los criterios para la administración perfecta, porque el gobierno carece de una organización unitaria dotada de una sola línea de autoridad sin ambigüedad, de reglas uniformes, de objetivos claramente definidos y jerarquizados, libre de presiones del tiempo, poseedora de una información perfecta y de consenso acerca de las metas entre las organizaciones" (Rose, 1999, p. 258). 
generales y concretas que acontecen en la realidad social, además, se ha caracterizado que las políticas públicas son las acciones que se priorizan en la sociedad para transformar las situaciones de necesidades y problemas, por tanto, se deben entender los procesos que operan en la escogencia de prioridades, ¿desde cuáles valores?, ¿qué papel juegan los actores?, ¿cuáles son los intereses?, ¿desde qué posición?, ¿se atiende realmente las necesidades con determinadas políticas públicas?, o si ¿las estrategias que escogen son los más adecuadas?, o si ¿existe una verdadera participación de las personas?

Es una tarea fundamental atender estas interrogantes de forma trans-disciplinar dentro de las Ciencias Sociales, desde perspectivas contextualizadas, que vayan más allá de la inmediatez del problema y busquen soluciones a través de enfoques integrales-transformadores, procurando, además, fomentar metodologías participativas que involucren a las personas en todas las etapas, desde la identificación de las necesidades, hasta la formulación, ejecución, monitoreo y evaluación de las políticas públicas, tareas que tanto la Sociología como la Ciencia Política articuladas con otras disciplinas pueden llevar a cabo, debido a la multiplicidad conceptual, teórica, y la gran variedad metodológica que las caracteriza, para analizar y trabajar sus intereses de estudio.

La evaluación orientada hacia la transformación en todo proceso de construcción de las políticas públicas, programas, proyectos, es necesaria para distanciarse de la acción, mejorar lo realizado, y obtener un proceso de aprendizaje y retroalimentación, que debe ser constante, siendo además una forma de control y rendición de cuentas con los gobernantes, por lo cual se debe promover, posicionado un rol activo de la ciudadanía o sociedad civil con sus deberes y derechos en relación con el papel del Estado, pero:

La reestructuración de la sociedad civil pasa, sin embargo, por una re-conceptualización del Estado de parte de los individuos, de sus prácticas, de sus responsabilidades, de su sentido de la organización social y de la forma de enfrentar o plantear el conflicto, lo cual no se logra fácilmente (Rivera, 1995, p. 123).

Aunado a lo anterior, la importancia de la evaluación desde el enfoque transformador, es su finalidad de modificar realidades, por medio, de la participación activa de las personas en la toma de decisiones sobre sus propios problemas, pero se debe dejar de lado posiciones tecnocráticas, verticales y tradicionales de la evaluación desde el enfoque positivista:

Es peligroso continuar con la práctica más común de la evaluación, aquella que se posiciona en un paradigma positivista, pues con ello se estaría consolidando el orden establecido. Con la evolución del pensamiento en las Ciencias Sociales se aclara la comprensión de la evaluación como proceso marcado por relaciones de poder y, por lo tanto, deja de ser un proceso ingenuo. Dentro de la dinámica de la globalización económica, la evaluación puede servir para legitimar procesos [...] que sirvan fundamentalmente a los intereses hegemónicos y por ello es necesario pensar alternativas para la evaluación desde enfoques más comprensivos y que busquen la posibilidad de la transformación (Vargas, 2001, p. 43).

A manera de cierre, la propuesta de principios para orientar la evaluación de las políticas públicas desde el rol transformador desde su planteamiento inicial, hasta su finalización, considera elementos primordiales e integrales, donde la evaluación es posicionada como una práctica situada y constante para mejorar el accionar y los resultados, ya que, algunas soluciones son temporales, al no esbozar claramente cuál es el origen de esas causas y no trabajar desde los actores (as), al ser así, se prioriza lo inmediato, esto es medidas paliativas, por lo que no es raro, siguiendo lo planteado por Rivera (1995) ver los mismos problemas una y otras vez cada año, lo que transforma en una reproducción cíclica del problema o necesidad, por consiguiente, se enfatiza la necesidad de conocer las causas estructurales, situando que es conocido las dificultades de encontrar intereses comunes y las limitantes de recursos, pero esto no debe eludir la elaboración de una acción pública transformadora. 


\section{Referencias}

Abarca, Allan. (2002). Las políticas públicas como perspectiva de análisis. Revista de Ciencias Sociales 97: 95-103, 2002 (III). Recuperado de http://revistacienciassociales.ucr.ac.cr/wp-content/ revistas/97/07-ABARCA_95-103.pdf

Ander-Egg, Ezequiel y Aguilar, María. (2004). Como elaborar un proyecto, Guía para diseñar proyectos sociales y culturales. Argentina, San Isidro Editorial LUMEN/HUMANITAS.

Aguilar, Luis. (1992). Estudio Introductorio, El estudio de las políticas públicas. México: Porrúa. Recuperado de http://www.inap.mx/portal/images/RAP/el\%20estudio\%20de\%20las\%20politicas\%20 publicas.pdf

Carmen, Raff. (2004). Desarrollo autónomo. Humanizar el paisaje: una incursión en el pensamiento y la práctica radicales. Heredia, Costa Rica. EUNA.

Cohen, Ernesto y Franco, Rolando (1988). Evaluación de Proyectos Sociales. Buenos Aires Argentina, Editores Gel.

Correa, Alfredo. (1998). Misión rural, una perspectiva regional. IICA-TM editores.

Costa, Armando. (2004). Planificación, monitoreo y evaluación-Proyectos de desarrollo social y humano. San José, Costa Rica Centro Cooperativo Sueco.

Gerston, Larry. (2010). Public Policy Making: Process and principles. United States [Políticas Públicas: Procesos y Principios]. M.E Sharpe, Inc.

González, Miguel. (2002). Las coordenadas básicas de la decisión racional. Entre la libertad del actor y el determinismo contextual. En Martinelli, José (coord.), Políticas públicas en el nuevo sexenio (pp-21-60). México: Plaza Valdés.

Gómez, Manuel y Sainz, Héctor. (2001). El ciclo del proyecto de cooperación al desarrollo: La aplicación del marco lógico. Madrid, España. Editorial Cideal.

Gómez, Carlos y Arango, Xochitl. (2012). Administración Pública y Gobernanza: El Papel de la gestión de la Ética en las Organizaciones Públicas. Daena: International Journal of Good Conscience. 7(3) 109-122. Recuperado de http://www.spentamexico.org/v7-n3/7\%283\%29109-122.pdf

Kuschick, Murillo (2002) Las encuestas de opinión como mecanismo para la evaluación de políticas públicas. En Martinelli, José (coord.), Políticas públicas en el nuevo sexenio (pp-105-119). México: Plaza Valdés.

Lahera, Eugenio. (2004). Política y políticas públicas. Serie políticas sociales. CEPAL.

Mayorga, Fernando, y Córdova, Eduardo. (2007). Gobernabilidad y Gobernanza en América latina. Working Paper NCCR Norte-Sur IP8, Ginebra. Recuperado de http://www.institut-gouvernance. org/docs/ficha-gobernabilida.pdf

Ministerio de Planificación Nacional y Política Económica (MIDEPLAN) (2014) Plan Nacional de Desarrollo 2015-2018 “Alberto Cañas Escalante" San José, Costa Rica. Recuperado de http:// extwprlegs1.fao.org/docs/pdf/cos145028.pdf

Meny, Ives y Jean Claude Thoening. (1992). Las políticas públicas. Barcelona. Ariel.

Nirenberg, Olga. (2003). Programación y evaluación de proyectos sociales: Aportes para la racionalidad. México, DF. Paidós

Nirenberg, Olga, Brawerman, Josette y Ruiz, Violeta. (2003). Programación y Evaluación de Proyectos Sociales: Aportes para la Racionalidad y Transparencia. Buenos Aires, Argentina. Editorial Paidós.

Smith, Catherine. (2005). Writing public policy: a practical guide to communicating in the policymaking process. [Redacción de políticas públicas: una guía práctica para la comunicación en el proceso de formulación de políticas] United States. Oxford University press.

Serna, José. (2010). Globalización y gobernanza: las transformaciones del Estado y sus implicaciones para el derecho público. Universidad Nacional Autónoma de México. Instituto de investigaciones jurídicas. Recuperado de http://biblio.juridicas.unam.mx/libros/6/2818/5.pdf 
Picado, Xinia. (2002). Criterios para realizar evaluaciones de calidad. Revista de Ciencias Sociales no 97, pág. 9-16. Recuperado de http://www.redalyc.org/pdf/153/15309702.pdf

Rose, Richard. (1999). El Gran Gobierno. Un acercamiento desde los programas gubernamentales. México, FCE.

Rivera, Roy. (1995). Descentralización y la metáfora de la Reforma del Estado. San José. FLACSO.

Rivera, Roy. (2002). El Contexto de la evaluación de proyectos sociales en el marco de una democracia deliberativa. Revista de Ciencias Sociales 97: 17-30, 2002 (III). Recuperado de http://www.revistacienciassociales.ucr.ac.cr/wp-content/revistas/97/02-RIVERA_17-30_.pdf

Ulloa, Luis y Giomi, Claudio. (2002). Guía para la elaboración de proyectos sociales. Colección liderazgo social No 5. Recuperado de https://www.ugto.mx/vinculacion/images/pdf/guiaproyectos.pdf

Uvalle, Ricardo. (2002). Los Fundamentos institucionales de la gestión pública. VII Congreso Internacional del CLAD sobre la Reforma del Estado y de la Administración Pública, Lisboa, Portugal, 8-11. Recuperado de http://unpan1.un.org/intradoc/groups/public/documents/CLAD/ clad0043511.pdf

Vargas, Alicia. (2001). Enfoques evaluativos, Revista de Ciencias Sociales. 92-93: 35-45. (II-III). Disponible en http://www.revistacienciassociales.ucr.ac.cr/wp-content/revistas/92-93/03-.pdf

\section{Notas: Citas textuales en versión original del idioma inglés}

1. "As problem solving activity, public policy making has three basic components: the problem, the policy, and the players" (Smith, 2005, p. 1).

2. "The most important characteristic of policy-making activity is that it is a process occurring in a context" (Smith, 2005, p.1).

3. "Like a barometer that reacts to different climatological conditions, the public agenda changes with shifting public priorities and values. As such, the public agenda assumes a dynamic presence" (Gerston, 2010, p. 48).

4. "Some problems are a "one -time" phenomenon: they reach the public agenda, are solved by policy makers, and never reappear. Other problems are open-ended in nature: they are not aggravating enough to capture support, yet sufficiently annoying to garner attention. Sometimes, an issue may be so complicated or so divisive that little other than temporary patchwork solutions can be applied, leaving the impression among participants that the issue will reemerge at another time" (Gerston, 2010, p. 48). 
